\title{
The experimental development of Ophiotaenia monnigi Fuhrmann, 1924 in Cyclops leuckarti
}

\author{
Gonghuang Cheng $\cdot$ Zaohe $\mathrm{Wu} \cdot$ Yuguang Lin
}

Published online: 5 February 2008

(C) Springer-Verlag 2008

Erratum to: Parasitol Res (2008)

DOI 10.1007/s00436-008-0875-x

The original version of this article unfortunately contained a mistake in author names. The correct version is given here.

\section{Gonghuang Cheng}

The online version of the original article can be found at http:// dx.doi.org/10.1007/s00436-008-0875-x

G. H. Cheng $(\bowtie) \cdot$ Z. H. Wu

Fisheries College, Guangdong Ocean University,

Zhanjiang 524025, China

e-mail: ghcheng@sohu.com

\section{Y. Lin}

Parasitology Laboratory, Xiamen University,

Xiamen, Fujian 361005, China

G. H. Cheng · Z. H. Wu

Guangdong Provincial Key Lab of Pathogenic Biology and Epidemiology for Aquatic Economic Animals,

Zhanjiang 524025, China 